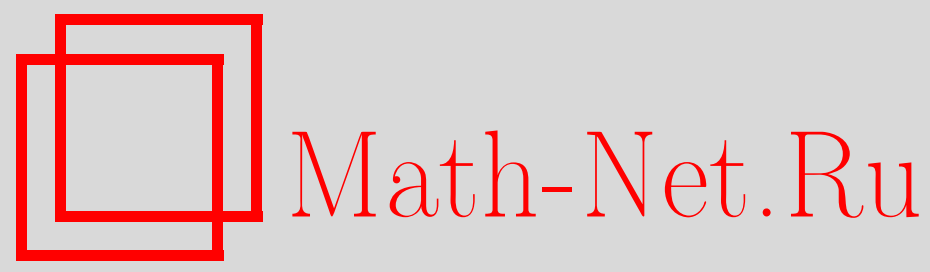

А. И. Павлов, Целые функции, аналитическое продолжение и дробные доли линейной функции, Матем. заметки, 1999, том 66, выпуск 4, 540-550

DOI: https://doi.org/10.4213/mzm3987

Использование Общероссийского математического портала Math-Net.Ru подразумевает, что вы прочитали и согласны с пользовательским соглашением http://www . mathnet.ru/rus/agreement

Параметры загрузки:

IP: 34.227 .88 .159

26 апреля 2023 г., $17: 48: 37$

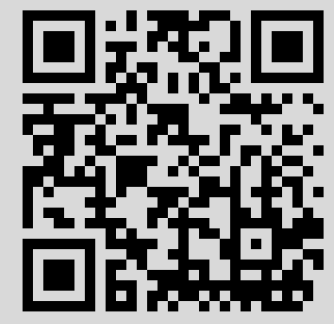




\section{ЦЕЛЫЕ ФУНКЦИИ, АНАЛИТИЧЕСКОЕ ПРОДОЛЖЕНИЕ И ДРОБНЫЕ ДОЛИ ЛИНЕЙНОЙ ФУНКЦИИ}

\section{А. И. Павлов}

Основньм результатом работы является

ТЕорема. Пусть челая функиия $G(z)$ удовлетворяет условиям:

1) тейлоровские коэффициенты функиии $G(z)$ неотрицательны;

2) для некоторых фиксированных $C>0 u A>0$ при $|z|>R_{0}$ выполнено

$$
|G(z)|<\exp \left(C \frac{|z|}{\ln ^{A}|z|}\right) .
$$

Пусть далее для некоторого фиксированного $\alpha>0$ отклонение $D_{N}$ последовательности $x_{n}=\{\alpha n\}, n=1,2, \ldots$, при $N \rightarrow \infty$ имеет оченку $D_{N}=$ $O\left(\ln ^{B} N / N\right)$. Тогда если функиия $G(z)$ не является тождественной постоянной и выполняется неравенство $B+1<A$, то степенной ряд $\sum_{n=0}^{\infty} G([\alpha n]) z^{n}$, сходящийся в круге $|z|<1$, не может быть аналитически продолжен в область $|z|>1$ через любую дугу на окружности $|z|=1$.

Библиографияя: 8 названий.

Введение. В 1899 году Вигерт доказал [1] следующее утверждение. Пусть $G(z)$ целая функция первого порядка минимального типа, т.е. для любого фиксированного $\varepsilon>0$ существует $R_{0}$ такое, что при $|z|>R_{0}$ вьполняется неравенство $|G(z)|<\exp (\varepsilon|z|)$. Тогда если $G(z) \not \equiv 0$, то степенной ряд $f(z)=\sum_{n=0}^{\infty} G(n) z^{n}$, сходящийся в круге $|z|<1$, аналитически продолжается на всю плоскость комплексного переменного $z$, кроме точки $z=1$, которая для функции $f(z)$ является либо полюсом, либо существенно особой тОчкой.

В работе [2] автор построил класс пар $(G, M)$ целых функций $G(z)$ и множеств $M$ натуральных чисел таких, что степенной ряд $\sum_{m \in M} G(m) z^{m}$, сходящийся в круге $|z|<1$, не может быть аналитически продолжен в область $|z|>1$ через любую дугу на окружности $|z|=1$. В частности, в работе [2] доказано следующее утверждение. Пусть целое $k \geqslant 2$ и $G(z)$ - целая трансцендентная функция, удовлетворяющая условиям:

1) тейлоровские коэффициенты функции $G(z)$ неотрицательны;

Работа выполнена при финансовой поддержке Российского фонда фундаментальных исследований, грант № 97-01-00580. 
2) функция $G(z)$ имеет порядок $\sigma<1-1 / k$ и нормальный тип, т.е. если $M(R)=$ $\max _{|z|=R}|G(z)|$, то

$$
\varlimsup_{R \rightarrow \infty} \frac{\ln M(R)}{R^{\sigma}}<\infty .
$$

Тогда если $M$ - множество всех натуральных чисел без $k$-х степеней, то степенной ряд $\sum_{m \in M} G(m) z^{m}$, сходящийся в круге $|z|<1$, имеет окружность $|z|=1$ своей гранищей аналитичности.

В 1921 году Гекке [3] доказал теорему: если $\alpha$ - действительное иррациональное число, то степенной ряд $\sum_{n=1}^{\infty}[\alpha n] z^{n}$, сходящийся в круге $|z|<1$, не может быть продолжен аналитически в область $|z|>1$ через любую дугу на окружности $|z|=1$. Здесь и далее $[x]$ - целая часть действительного числа $x$, т.е. наибольшее среди тех целых $m$, для которых выполняется неравенство $m \leqslant x$.

В 1962 году Шварц [4] методом Гекке доказал аналогичное утверждение для ряда $\sum_{n=1}^{\infty} P([\alpha n]) z^{n}$, где $P(z)$ - произвольньй многочлен положительной степени.

В 1965 году американские математики Кэролл и Кемпермен [5] доказали аналитическую непродолжаемость в область $|z|>1$ ряда $\sum_{n=1}^{\infty} G([\alpha n]) z^{n}$, где $\alpha$ - действительное иррациональное число, а

$$
G(z)=\sum_{\nu=1}^{k} C_{\nu}(\ln z)^{\rho_{\nu}} z^{\sigma_{\nu}} \exp \left(d_{\nu} z^{\tau_{\nu}}\right),
$$

где $\rho_{\nu}, \sigma_{\nu}, d_{\nu}$ и $\tau_{\nu}$ - действительные постоянные, $\tau_{\nu}<1, C_{\nu}$ - любые комплексные числа.

Прежде чем формулировать основные результаты работы, напомним одно определение. Пусть $x_{n}, n=1,2, \ldots,-$ произвольная последовательность действительных чисел с условием $0 \leqslant x_{n}<1$. Тогда если

$$
V(\gamma, N)=\sum_{\substack{n \leqslant N \\ x_{n}<\gamma}} 1, \quad 0 \leqslant \gamma<1,
$$

то по определению отклонением последовательности $x_{n}$ называется набор чисел

$$
D_{N}=\sup _{0 \leqslant \gamma<1}\left|\frac{V(\gamma, N)}{N}-\gamma\right|, \quad N \geqslant 1 .
$$

В работе будут доказаны следующие утверждения.

ТЕорема. Пусть иелая функиия $G(z)$ удовлетворяет условиям:

1) тейлоровские коэффиииенты функиии $G(z)$ неотрицательны;

2) существуют постоянные $C_{0}>0$ и $A>0$ такие, что

$$
|G(z)|<\exp \left(C_{0} \frac{|z|}{\ln ^{A}|z|}\right), \quad|z|>R_{0} .
$$


Пусть далее фиксированное $\alpha \in(0,1)$ u $D_{N}$ - отклонение последовательности $x_{n}=\{\alpha n\}$, где $\{t\}-$ дробная часть числа $t$. Тогда если

$$
D_{N}=O\left(\frac{\ln ^{B} N}{N}\right), \quad N \rightarrow \infty,
$$

әде постоянная $B \geqslant 1$, причем $B+1<A, u G(z) \not \equiv C$, то степенной ряд $\sum_{n=1}^{\infty} G([\alpha n]) z^{n}$, сходящийся в круге $|z|<1$, не может быть аналитически продолэсен в область $|z|>1$ через любую дугу на окружности $|z|=1$.

СлЕДСТВИЕ 1. Если положительное число $\alpha$ при разложении в чепную дробь имеет ограниченные неполные частные и челая функиия $G(z)$ удовлетворяет условиям теоремы с постоянной $A>2$, то имеет место утвержсдение теоремы.

СледствИЕ 2. Если целая функиия $G(z)$ удовлетворяет условиям теоремы $c$ постоянной $A>3$, то для почти всех $\alpha \in(0,1)$ по мере Лебега выполняется утверждение теоремы.

Прежде чем приступить к доказательству сформулированных утверждений, сделаем два замечания.

ЗАмЕчАниЕ 1. Если $G(z)$ - многочлен, то требование неотрицательности коэффициентов этого многочлена можно отбросить.

ЗАМЕчАнИЕ 2 . Если $\alpha$ является рациональным числом $\alpha=a / q$, где целые $a$ и $q$ удовлетворяют условиям $1 \leqslant a \leqslant q-1$ и $(a, q)=1$, и $G(z)$ - произвольная целая функция первого порядка минимального типа, то, как нетрудно доказать, при условии $G(z) \not \equiv C$ степенной ряд $f(z)=\sum_{n=0}^{\infty} G([\alpha n]) z^{n}$, сходящийся в круге $|z|<1$, может быть аналитически продолжен на всю комплексную плоскость, кроме точек $z_{k}=e^{2 \pi i k / q}, k=1$, $2, \ldots, q$, каждая из которых для функции $f(z)$ является изолированной особой точкой однозначного характера.

\section{1. Леммы.}

ЛЕмма 1. Если иелая функиия

$$
G(z)=\sum_{n=0}^{\infty} \frac{a_{n}}{n !} z^{n}
$$

удовлетворяет условию

$$
|G(z)|<\exp \left(C \frac{|z|}{\ln ^{A}|z|}\right),
$$

әде $C$ и $A$ - полохительные постоянные, $|z|>R_{0}, u$ если

$$
g(z)=\sum_{n=0}^{\infty} \frac{a_{n}}{z^{n+1}}
$$

mo npu

$$
M(R)=\max _{|z|=R}\left|g\left(\frac{1}{z}\right)\right|
$$

выполняется оценка $\ln \ln M(R)=O\left(R^{1 / A}\right), R \rightarrow \infty$. 
ДокАЗАТЕЛЬСТво. Известно [6], что для любого $\varphi_{0} \in[0,2 \pi]$ выполняется равенство

$$
g(z)=\int_{0}^{\infty} G\left(t e^{-i \varphi_{0}}\right) \exp \left(-z t e^{-i \varphi_{0}}-i \varphi_{0}\right) d t .
$$

Поэтому если $\varphi_{0}=\arg z$ и $|z|=1 / R$, то

$$
g\left(\frac{e^{i \varphi}}{R}\right)=\int_{0}^{\infty} G\left(t e^{-i \varphi_{0}}\right) \exp \left(-\frac{t}{R}-i \varphi_{0}\right) d t
$$

или

$$
M(R)=O\left(\int_{0}^{\infty} \exp \left(C \frac{t}{\ln ^{A} t}-\frac{t}{R}\right) d t\right) .
$$

Пусть $t_{R}$ определено так, что при $t \geqslant t_{R}$ вьполняется неравенство

$$
C \frac{t}{\ln ^{A} t}-\frac{t}{R}<-\frac{t}{2 R}
$$

Тогда

$$
M(R)=O\left(\int_{0}^{t_{R}} \exp \left(\frac{C t}{\ln ^{A} t}\right) d t+\int_{t_{R}}^{\infty} \exp \left(-\frac{t}{2 R}\right) d t\right)=O\left(t_{R} \exp \left(\frac{C t_{R}}{\ln ^{A} t_{R}}\right)+2 R\right) .
$$

Легко видеть, что $t_{R}=\exp \left((2 C R)^{1 / A}\right)$. Значит, $\ln \ln M(R)=O\left(R^{1 / A}\right)$. Лемма 1 доказана.

Лемма 2 (Коксма [7]). Пусть $D_{N}$ - отклонение последовательности $\{\alpha n\}$, $n=1,2, \ldots, u f(x)-$ комплекснозначная функиия, непрерьвно дифференцируемая на отрезке $[0,1]$. Тогда

$$
\sum_{n=1}^{N} f(\{\alpha n\})-N \int_{0}^{1} f(x) d x=O\left(N D_{N} \int_{0}^{1}\left|f^{\prime}(x)\right| d x\right), \quad N \rightarrow \infty .
$$

ЛЕмма 3. Пусть

$$
S(R)=\int_{|t|=\rho} \frac{\left|1-r e^{\alpha t}\right|}{1-r e^{\alpha \operatorname{Re} t}} d t
$$

әде

$$
0<r<1, \quad \frac{1}{R}=\ln \frac{1}{r}, \quad \rho=\frac{1}{\alpha R}\left(1-\frac{1}{T(R)}\right),
$$

$T(R)$ - произвольная непрерывная возрастающая функиия от $R$ с условием

$$
\lim _{R \rightarrow \infty} T(R)=\infty
$$

Тогда

$$
S(R)=O\left(\frac{\ln T(R)}{R}\right), \quad R \rightarrow \infty .
$$


ДоКАЗАТЕЛЬСТво. Пусть

$$
f_{0}(\varphi)=\frac{\left|1-r \exp \left(\alpha \rho e^{i \varphi}\right)\right|}{1-r \exp (\alpha \rho \cos \varphi)}
$$

Тогда

$$
f_{0}(\varphi)=\frac{\left|1-\exp \left(\alpha \rho e^{i \varphi}-1 / R\right)\right|}{1-\exp (\alpha \rho \cos \varphi-1 / R)}
$$

Ясно, что при $R \rightarrow \infty$

$$
\begin{aligned}
1-\exp \left(\alpha \rho e^{i \varphi}-\frac{1}{R}\right) & =1-\exp \left(\alpha \rho \cos \varphi-\frac{1}{R}\right)(\cos (\alpha \rho \sin \varphi)+i \sin (\alpha \rho \sin \varphi)) \\
& =1-\exp \left(\alpha \rho \cos \varphi-\frac{1}{R}\right)\left(1+O\left(\frac{\sin ^{2} \varphi}{R^{2}}\right)\right)+O\left(\frac{|\sin \varphi|}{R}\right) \\
& =1-\exp \left(\alpha \rho \cos \varphi-\frac{1}{R}\right)+O\left(\frac{|\sin \varphi|}{R}\right) .
\end{aligned}
$$

Если $x \rightarrow 0$, то $1-e^{-x}=x+O\left(x^{2}\right)$. Поэтому

$$
\begin{aligned}
1-\exp \left(\alpha \rho \cos \varphi-\frac{1}{R}\right) & =\frac{1}{R}-\alpha \rho \cos \varphi+O\left(\left(\frac{1}{R}-\alpha \rho \cos \varphi\right)^{2}\right) \\
& =\frac{1}{R}\left(2 \sin ^{2} \frac{\varphi}{2}+\frac{\cos \varphi}{T(R)}\right)+O\left(R^{-2}\left(\sin ^{2} \frac{\varphi}{2}+\frac{1}{T(R)}\right)^{2}\right) .
\end{aligned}
$$

Пусть $\varphi_{0}=\varphi_{0}(R)=(\ln T(R))^{-1 / 2}$. Тогда если $|\varphi| \leqslant \varphi_{0}$, то при $R \rightarrow \infty$ получим

$$
\begin{aligned}
f_{0}(\varphi) & =1+\frac{O(|\sin \varphi|)}{2 \sin ^{2} \frac{\varphi}{2}+\frac{\cos \varphi}{T(R)}+O\left(R^{-1}\left(\sin ^{4} \frac{\varphi}{2}+(T(R))^{-2}\right)\right)} \\
& =1+O\left(\frac{|\sin \varphi|}{\sin ^{2} \frac{\varphi}{2}+(T(R))^{-1}}\right) .
\end{aligned}
$$

Следовательно,

$$
\begin{aligned}
\int_{-\varphi_{0}}^{\varphi_{0}} f_{0}(\varphi) d \varphi & =O\left(\varphi_{0}+\int_{0}^{\varphi_{0}} \frac{\varphi T(R) d \varphi}{\varphi^{2} T(R)+1}\right) \\
& =O\left((\ln T(R))^{-1 / 2}+\ln \left(1+\varphi_{0}^{2} T(R)\right)\right)=O(\ln T(R)), \quad R \rightarrow \infty .
\end{aligned}
$$

Если $\varphi_{0} \leqslant|\varphi| \leqslant \pi$, то

$$
f_{0}(\varphi)=1+O\left(\frac{1}{\sin ^{2} \frac{\varphi}{2}-(T(R))^{-1}}\right)=1+O\left(\frac{1}{\sin ^{2} \frac{\varphi_{0}}{2}-(T(R))^{-1}}\right)=O\left(\varphi_{0}^{-2}\right) .
$$

Поэтому

$$
\int_{\varphi_{0} \leqslant|\varphi| \leqslant \pi} f_{0}(\varphi) d \varphi=O\left(\varphi_{0}^{-2}\right)=O(\ln T(R)), \quad R \rightarrow \infty .
$$

Лемма 3 доказана, так как

$$
S(R)=O\left(R^{-1} \int_{0}^{2 \pi} f_{0}(\varphi) d \varphi\right) .
$$


ЛЕмма 4. Пусть

$$
\psi(r, t)=\frac{g(t)\left(1-e^{-t}\right)}{(2 \pi i m-t)\left(1-r e^{\alpha t}\right)},
$$

где $g(t)$ - функиия из леммы 1 , иелое $m \neq 0$ u

$$
\rho=\frac{1}{\alpha R}\left(1-\frac{1}{T(R)}\right) \quad R=\ln \frac{1}{r}, \quad T(R)=(\ln M(R))^{C},
$$

постоянная $C>0,0<r<1,0<\alpha<1$,

$$
M(R)=\max _{|t|=R}\left|g\left(\frac{1}{t}\right)\right| .
$$

Тогда при $r \rightarrow 1$ выполняется равенство

$$
I(r)=\frac{1}{2 \pi i} \int_{|t|=\rho} \psi(r, t) d t=\frac{1-r}{2 \pi i \alpha^{2} m} g\left(\frac{1}{\alpha} \ln \frac{1}{r}\right)(1+O(1-r)) .
$$

ДокАЗАТЕЛЬСтво. Пусть $\Gamma=\{t:|t|=1\}$. Так как функция $\psi(r, t)$ в кольце $\rho<|t|<1$ имеет только одну особую точку $t=\frac{1}{\alpha} \ln \frac{1}{r}$, являющуюся простым полюсом, то по теореме Коши

$$
I(r)=\frac{1}{2 \pi i} \int_{\Gamma} \psi(r, t) d t-\operatorname{res}\left(\psi(r, t), \frac{1}{\alpha} \ln \frac{1}{r}\right) .
$$

Легко видеть, что

$$
\begin{aligned}
\operatorname{res}\left(\psi(r, t), \frac{1}{\alpha} \ln \frac{1}{r}\right) & =-\frac{1-\exp \left(-\frac{1}{\alpha} \ln \frac{1}{r}\right)}{\alpha\left(2 \pi i m-\frac{1}{\alpha} \ln \frac{1}{r}\right)} g\left(\frac{1}{\alpha} \ln \frac{1}{r}\right) \\
& =-\frac{1-r}{2 \pi i \alpha^{2} m} g\left(\frac{1}{\alpha} \ln \frac{1}{r}\right)(1+O(1-r))
\end{aligned}
$$

Покажем, что при $r \rightarrow 1$ выполняется равенство

$$
\int_{\Gamma} \psi(r, t) d t=O(1)
$$

Действительно, если $|t|=1$, то при $r \rightarrow 1$ получим

$$
\frac{\left(1-e^{-t}\right) g(t)}{2 \pi i m-t}=O(1) .
$$

Далее, функция $1-r e^{\alpha t}$ имеет нули в точках

$$
t_{k}=\frac{1}{\alpha}\left(\ln \frac{1}{r}+2 k \pi i\right), \quad k=0, \pm 1, \pm 2, \ldots .
$$

Поэтому при $|t|=1$ получим

$$
\varliminf_{r \rightarrow 1}\left|1-r e^{\alpha t}\right|>0 .
$$

Лемма 4 доказана. 
ЛЕмма 5 [8]. Пусть функиии $V(x)$ и $\mu(x)$ определены на интервале $(0, \infty)$, непрерывны, положительны, возрастают и

$$
\int_{1}^{\infty} \frac{d x}{\mu(x)}<\infty
$$

Тогда для любого фиксированного $h>0$ мнохество

$$
E_{h}=\left\{x: V\left(x+\frac{1}{\mu(V(x))}\right)>h+V(x)\right\}
$$

имеет конечную меру.

ЛЕмма 6. Если $f(z)-$ иелая функиия $и$

$$
M(R)=\max _{|z|=R}|f(z)|,
$$

то для любого є >0 существуют постоянная $C>0$ и последовательность $R_{k}$, $k=1,2, \ldots$, действительных чисел такие, что

$$
\lim _{k \rightarrow \infty} R_{k}=\infty
$$

и выполняется неравенство

$$
M\left(R_{k}+\frac{R_{k}}{\left(\ln M\left(R_{k}\right)\right)^{1+\varepsilon}}\right)<C M\left(R_{k}\right) .
$$

ДокАЗАТЕльСТво. Если $\ln M\left(e^{x}\right)=V(x)$, то согласно лемме 5 для любых фиксированных $\beta>1$ и $h>0$ существуют действительные $x_{k}$ такие, что

$$
\lim _{k \rightarrow \infty} x_{k}=\infty \quad \text { и } \quad V\left(x_{k}+\frac{1}{\left(V\left(x_{k}\right)\right)^{\beta}}\right)<h+V\left(x_{k}\right) .
$$

Следовательно,

$$
\ln M\left(\exp \left(x_{k}+\frac{1}{\left(\ln M\left(e^{x_{k}}\right)\right)^{\beta}}\right)\right)<h+\ln M\left(e^{x_{k}}\right) .
$$

Пусть $R_{k}=e^{x_{k}}$ и $\beta=1+\varepsilon$. Тогда

$$
\begin{aligned}
\ln M\left(R_{k}+\frac{R_{k}}{\left(\ln M\left(R_{k}\right)\right)^{1+\varepsilon}}\right) & <\ln M\left(R_{k} \exp \left(\frac{1}{\left(\ln M\left(R_{k}\right)\right)^{1+\varepsilon}}\right)\right) \\
& =\ln M\left(\exp \left(x_{k}+\frac{1}{\left(\ln M\left(e^{x_{k}}\right)\right)^{1+\varepsilon}}\right)\right)<h+\ln M\left(R_{k}\right) .
\end{aligned}
$$

Поэтому

$$
M\left(R_{k}+\frac{R_{k}}{\left(\ln M\left(R_{k}\right)\right)^{1+\varepsilon}}\right)<C M\left(R_{k}\right), \quad C=e^{h} .
$$

Лемма 6 доказана. 
2. Доказательства теоремы и следствий. Если

$$
G(z)=\sum_{n=0}^{\infty} \frac{a_{n}}{n !} z^{n}, \quad g(z)=\sum_{n=0}^{\infty} \frac{a_{n}}{z^{n+1}}
$$

то, как известно [6], при любом фиксированном $\rho>0$

$$
G(z)=\frac{1}{2 \pi i} \int_{|t|=\rho} g(t) e^{z t} d t
$$

Следовательно, если $|z|<1$ и

$$
F_{1}(z)=\sum_{n=0}^{\infty} G(\alpha n) z^{n}, \quad F_{2}(z)=\sum_{n=0}^{\infty} G([\alpha n]) z^{n}
$$

то при $F(z)=F_{1}(z)-F_{2}(z)$ вьполняется равенство

$$
F(z)=\frac{1}{2 \pi i} \int_{|t|=\rho} g(t) \Phi(z, t) d t
$$

где

$$
\Phi(z, t)=\sum_{n=0}^{\infty}\left(1-e^{-t\{\alpha n\}}\right)\left(z e^{\alpha t}\right)^{n} .
$$

Пусть $\rho$ настолько мало, что $\left|z e^{\alpha t}\right|=|z| e^{\alpha \operatorname{Re} t}<1$ и $z=r e^{2 \pi i \alpha m}$, где целое $m \neq 0$ фиксировано, $0<r<1$. Согласно теореме Вигерта существует целая функция $F_{0}(z)$ такая, что вьполняется равенство

$$
F_{1}(z)=F_{0}\left(\frac{1}{1-z}\right)
$$

Значит, для доказательства теоремы достаточно обосновать, что при любом фиксированном целом $m \neq 0$ имеет место асимптотика

$$
F\left(r e^{2 \pi i \alpha m}\right) \sim \frac{1-r}{2 \pi i \alpha^{2} m} g\left(\frac{1}{\alpha} \ln \frac{1}{r}\right), \quad r \rightarrow 1 .
$$

Пусть для комплексного $t$ и целого $N \geqslant 0$

$$
S_{N}(t)=\sum_{n=0}^{N}\left(1-e^{-t\{\alpha n\}}\right) e^{2 \pi i \alpha m n} .
$$

Тогда

$$
\Phi\left(r e^{2 \pi i \alpha m}\right)=\left(1-r e^{\alpha t}\right) \sum_{N=0}^{\infty} S_{N}(t)\left(r e^{\alpha t}\right)^{N}
$$


Далее, если $f(x, t)=\left(1-e^{-t x}\right) e^{2 \pi i m x}$, где $0 \leqslant x \leqslant 1$, то согласно лемме 2

$$
\sum_{n=0}^{N} f(\{\alpha n\}, t)-N \int_{0}^{1} f(x, t) d x=O\left(N D_{N} \int_{0}^{1}\left|\frac{\partial f}{\partial x}\right| d x\right)
$$

Так как для любого целого $m \neq 0$ вьполняется равенство

$$
\int_{0}^{1} f(x, t) d x=\frac{1-e^{-t}}{2 \pi i m-t}
$$

и равномерно по $x \in[0,1]$ при $|t| \leqslant \rho$ имеет место оценка $\partial f / \partial x=O(|t|)$, то согласно $(4)$

$$
S_{N}(t)=N \frac{1-e^{-t}}{2 \pi i m-t}+O\left(|t| N D_{N}\right)
$$

Следовательно, пользуясь условием (1) теоремы, из (5) получим

$$
\begin{aligned}
\Phi\left(r e^{2 \pi i \alpha m}\right)= & \frac{\left(1-r e^{\alpha t}\right)\left(1-e^{-t}\right)}{2 \pi i m-t} \sum_{N=1}^{\infty} N\left(r e^{\alpha t}\right)^{N} \\
& +O\left(|t|\left(1-r e^{\alpha t}\right) \sum_{N=1}^{\infty} \ln ^{B} N\left(r e^{\alpha t}\right)^{N}\right) \\
= & \frac{1-e^{-t}}{(2 \pi i m-t)\left(1-r e^{\alpha t}\right)} \\
& +O\left(\frac{\left|1-e^{-t}\right|}{|2 \pi i m-t|}+|t| \cdot\left|1-r e^{\alpha t}\right| \sum_{N=1}^{\infty} \ln ^{B} N\left(r\left|e^{\alpha t}\right|\right)^{N}\right) .
\end{aligned}
$$

Легко видеть, что при фиксированном $B>0$ и $r \rightarrow 1$

$$
\sum_{N=1}^{\infty} \ln ^{B} N\left(r\left|e^{\alpha t}\right|\right)^{N}=O\left(\frac{1}{1-r e^{\alpha \operatorname{Re} t}} \ln ^{B} \frac{1}{1-r e^{\alpha \operatorname{Re} t}}\right)
$$

Пусть

$$
M(R)=\max _{|z|=R}\left|g\left(\frac{1}{z}\right)\right|, \quad \rho=\frac{1}{\alpha R}\left(1-\frac{1}{\ln ^{C} M(R)}\right), \quad \frac{1}{R}=\ln \frac{1}{r}, \quad 0<r<1,
$$

где $C>1$ и фиксировано. Тогда при $\rho=|t|$ получим

$$
1-r e^{\alpha \operatorname{Re} t} \geqslant 1-r e^{\alpha \rho}=1-\exp \left(-\left(R \ln ^{C} M(R)\right)^{-1}\right)>\frac{1}{2}\left(R \ln ^{C} M(R)\right)^{-1} \text {. }
$$

Значит,

$$
\ln \frac{1}{1-r e^{\alpha \operatorname{Re} t}}=O(\ln \ln M(R)), \quad R \rightarrow \infty
$$


Следовательно, согласно (7)

$$
\sum_{N=1}^{\infty} \ln ^{B} N\left(r e^{\alpha \operatorname{Re} t}\right)^{N}=O\left(\frac{(\ln \ln M(R))^{B}}{1-r e^{\alpha \operatorname{Re} t}}\right)
$$

Пользуясь (6) и (8), получим

$$
\Phi\left(r e^{2 \pi i \alpha m}\right)=\frac{1-e^{-t}}{(2 \pi i m-t)\left(1-r e^{\alpha t}\right)}+O\left(|t|+|t| \frac{\left|1-r e^{\alpha t}\right|}{1-r e^{\alpha \operatorname{Re} t}}(\ln \ln M(R))^{B}\right) .
$$

Подставляя полученное равенство в (2), согласно лемме 3 имеем

$$
F\left(r e^{2 \pi i \alpha m}\right)=\frac{1}{2 \pi i} \int_{|t|=\rho} \frac{g(t)\left(1-e^{-t}\right)}{(2 \pi i m-t)\left(1-r e^{\alpha t}\right)} d t+O\left(\rho^{2} M\left(\frac{1}{\rho}\right)(\ln \ln M(R))^{B+1}\right) .
$$

Согласно лемме 4 при $r \rightarrow 1$

$$
I(r) \stackrel{\text { def }}{=} \frac{1}{2 \pi i} \int_{|t|=\rho} \frac{g(t)\left(1-e^{-t}\right)}{(2 \pi i m-t)\left(1-r e^{\alpha t}\right)} d t \sim \frac{1-r}{2 \pi i \alpha^{2} m} g\left(\frac{1}{\alpha} \ln \frac{1}{r}\right) .
$$

Так как тейлоровские коэффициенты функции $g(z)$ неотрицательны, то

$$
g\left(\frac{1}{\alpha} \ln \frac{1}{r}\right)=M(\alpha R)
$$

Таким образом, из (10) следует асимптотика

$$
I(r) \sim \frac{1-r}{2 \pi i \alpha^{2} m} M(\alpha R), \quad r \rightarrow 1 .
$$

Согласно выбору $\rho$ имеем

$$
M\left(\frac{1}{\rho}\right)=O\left(M\left(\alpha R+\frac{2 \alpha R}{\ln ^{C} M(R)}\right)\right) .
$$

Значит, согласно лемме 2 из (9) и (11) получим

$$
F\left(r e^{2 \pi i \alpha m}\right)=(1+o(1)) \frac{1-r}{2 \pi i \alpha^{2} m}+O\left(R^{(B+1) / A-2} M\left(\alpha R+\frac{2 \alpha R}{\ln ^{C} M(R)}\right)\right) .
$$

Напомним, что при $r \rightarrow 1$ имеем

$$
\frac{1}{R}=\frac{1}{\alpha} \ln \frac{1}{r} \sim \frac{1-r}{\alpha} .
$$

Согласно лемме 5 существует последовательность $R_{k}, k=1,2, \ldots$, такая, что

$$
\lim _{k \rightarrow \infty} R_{k}=\infty \quad \text { и } \quad M\left(\alpha R_{k}+\frac{2 \alpha R_{k}}{\ln ^{C} M\left(R_{k}\right)}\right)=O\left(M\left(\alpha R_{k}\right)\right), \quad k \rightarrow \infty .
$$


Следовательно, пользуясь неравенством $B+1<A$ и равенством $(12)$, получим

$F\left(r e^{2 \pi i \alpha m}\right)=\frac{1+o(1)}{2 \pi i \alpha^{2} m R_{k}} M\left(\alpha R_{k}\right)+O\left(R_{k}^{(B+1) / A-2} M\left(\alpha R_{k}\right)\right) \sim \frac{M\left(\alpha R_{k}\right)}{2 \pi i \alpha^{2} m R_{k}}, \quad k \rightarrow \infty$.

Из условия $G(z) \not \equiv C$ следует

$$
\lim _{k \rightarrow \infty} M\left(\alpha R_{k}\right)=\infty
$$

Значит, равенство (3), а вместе с ним и теорема доказаны.

Для доказательства следствия 1 достаточно заметить, что если положительное $\alpha$ при разложении в цепную дробь имеет ограниченные неполные частные, то отклонение $D_{N}$ последовательности $x_{n}=\{\alpha n\}, n=1,2, \ldots$, при $N \rightarrow \infty$ имеет оценку $D_{N}=$ $O(\ln N / N)$. Аналогично, для доказательства следствия 2 достаточно заметить, что для почти всех $\alpha \in[0,1]$ отклонение $D_{N}$ указанной последовательности при $N \rightarrow \infty$ имеет оценку $D_{N}=O\left(\ln ^{2+\varepsilon} N / N\right)$ для любого фиксированного $\varepsilon>0$.

\section{СПИСОК ЦИТИРОВАННОЙ ЛИТЕРАТУРЫ}

[1] Wigert S. Sur les fonction entières // Oefversigt af K. Vet. Ak. Forh. 1900. V. 57. P. 1001-1011.

[2] Павлов А.И. О некоторых классах степенных рядов, аналитически непродолжимых за свой круг сходимости // Изв. РАН. Сер. матем. 1997. Т. 61. № 4. С. 119-136.

[3] Hecke E. Über analytische Funktionen und die Verteilung von Zahlen mod Eins // Abh. Math. Sem. Univ. Hamburg. 1922. V. 1. P. 54-76.

[4] Schwarz W. Irrazionale Potezreihen // Arch. Math. (Basel). 1962. V. 13. №1-3. P. 228-240.

[5] Caroll F.W., Kempermen J.H. Noncontinuable analytic functions // Duke Math. J. 1965. V. 32. P. $65-84$.

[6] Гельфонд А. О. Исчисление конечных разностей. М.: Физматгиз, 1959.

[7] Koksma J.F. Een algemeene stelling uit de theorie der gelijkmatige verdeeling modulo $1 / /$ Mathematika B (Zutphen). 1942-43. V. 11. P. 7-11.

[8] Kovari T. A gap-theorem for entire function of infinite order // Michigan Math. J. 1965. V. 12. P. 133-140. 